\title{
Ist der Berlin-Fragebogen ein geeignetes Instrument der schlafmedizinischen Diagnostik in der pneumologischen Rehabilitation?
}

\author{
Is the Berlin Questionnaire an Appropriate Diagnostic Tool \\ for Sleep Medicine in Pneumological Rehabilitation?
}

Autoren

Institute
G. Weinreich ${ }^{1}$, K. Plein ${ }^{2}$, T. Teschler ${ }^{1}$, J. Resler ${ }^{2}$, H. Teschler ${ }^{1}$

Ruhrlandklinik, Abteilung Pneumologie - Universitätsklinik, Essen

Nordseeklinik Borkum, Borkum eingereicht 7.42006

akzeptiert 12.72006

\section{Bibliografie}

DOI $10.1055 / \mathrm{s}-2006-944270$

Pneumologie 2006; 60; 737-742

(c) Georg Thieme Verlag KG

Stuttgart · New York

ISSN 0934-8387

\section{Korrespondenzadresse}

Prof. Dr. med. Helmut Teschler Ruhrlandklinik

Tüschener Weg 40

45239 Essen

helmut.teschler@ruhrlandklinik.de

\section{Zusammenfassung}

Hintergrund: Der Berlin-Fragebogen ist ein diagnostisches Mittel, um Patienten mit einem hohen Risiko für eine obstruktive Schlafapnoe (OSA) zu ermitteln. Die Beurteilung basiert auf 13 Fragen zu den wichtigsten SBAS-Symptomen: Schnarchverhalten und Atempausen, Tagesschläfrigkeit, Bluthochdruck und Übergewicht.

Methoden: An 153 Rehabilitationspatienten mit Atemwegserkrankungen wurde ein nächtliches Schlafapnoescreening mit ApneaLink ${ }^{\circledR}$ (ResMed, München) durchgeführt und der RespiratoryDisturbance-Index (RDI) ermittelt, um die Sensitivität und Spezifität des Berlin-Fragebogens für eine OSA zu bestimmen. Dieser wurde den Studienteilnehmern zusammen mit der EpworthSchläfrigkeitsskala (ESS) ausgehändigt. Die Resultate wurden mit publizierten Daten hinsichtlich eines Referenzkollektivs verglichen, das sich in Allgemeinkrankenhäusern oder bei Allgemeinmedizinern in Behandlung befand.

Ergebnisse: Für das Studienkollektiv ergab sich bei einem Grenzwert von 10/h für den RDI eine Sensitivität von $62,5 \%$ und eine Spezifität von $52,8 \%$. Der positive Vorhersagewert betrug $38,4 \%$, der negative $74,2 \%$. Der positive Likelihood-Quotient wies einen Wert von 1,35 auf und der negative einen Wert von 0,69. Bei einem Grenzwert von 15/h betrug die Sensitivität 67,2\% und die Spezifität 53,8\%. Für diesen RDI-Wert lag der positive Vorhersagewert bei $25,1 \%$ und der negative bei $84,7 \%$. Der positive Likelihood-Quotient wies einen Wert von 1,42 auf und der negative einen Wert von 0,62. Im Studienkollektiv fanden sich Hinweise auf eine vermehrte Tagesschläfrigkeit ( $E S S=8,8 \pm 4,8$ ). Alle Symptome und Risikofaktoren traten bei den Rehabilitationspatienten signifikant häufiger auf als beim Referenzkollektiv. Gemäß des Berlin-Fragebogens waren Schnarchen und Atempausen bei Männern um 6,7\% häufiger anzutreffen, bei Frau-

\section{Abstract}

Background: The Berlin Questionnaire is an explorative tool of 13 questions designed to identify patients with obstructive sleep apnea. The questions are targeted toward key symptoms of snoring, apneas, daytime sleepiness, hypertension and overweight.

Methods: 153 patients undergoing pulmonary rehabilitation were screened for obstructive sleep apnea via i) ApneaLink ${ }^{\circledR}$ (ResMed, Munich) and ii) the Berlin Questionnaire. The Epworth Sleepiness Scale (ESS) was administered to grade daytime sleepiness. Results of ApneaLink ${ }^{\circledR}$ screening and questionnaires were prospectively compared. The Respiratory Disturbance Index (RDI) was used to compute predictive performance of the Berlin Questionnaire for obstructive sleep apnea. Results were also compared to previously published data from a primary care patient cohort.

Results: At a cut-off RDI $=10 / \mathrm{h}$, the Berlin Questionnaire had a sensitivity of $62.5 \%$, a specificity of $53.8 \%$, a positive predictive value of $38.4 \%$, a negative predictive value of $74.2 \%$, a positive likelihood ratio of 1.35 and a negative likelihood ratio of 0.69 . At a cut-off $R D I=15 / h$, the Berlin Questionnaire had a sensitivity of $67.2 \%$, a specificity of $52.8 \%$, a positive predictive value of $25.1 \%$, a negative predictive value of $84.7 \%$, a positive likelihood ratio of 1.42 and a negative likelihood ratio of 0.62 . There was evidence that the participants of this study suffered from an increased daytime sleepiness (ESS $=8.8 \pm 4.8$ ). The study group was more likely to snore, have apneas (men $6.7 \%$ more often, women $12.6 \%$ more often) and suffer from daytime sleepiness (men 19.0\% more often, women $14.6 \%$ more often) than the control group. Also, hypertension and overweight were more prevalent (men $6.1 \%$ more often, women $11.1 \%$ more often). 
en um 12,6\%. Das Symptom Tagesschläfrigkeit kam bei Männern um 19,0\% häufiger vor, bei Frauen um 14,6\%. Bluthochdruck und Übergewicht waren bei Männern um 6,1\% häufiger, bei Frauen um $11,1 \%$.

Schlussfolgerung: Der Berlin-Fragebogen besitzt bei Patienten in der pneumologischen Rehabilitation keine hinreichende Aussagekraft, um Patienten mit einem hohen Risiko für das Vorliegen einer obstruktiven Schlafapnoe zu ermitteln.

\section{Einleitung}

Schlafbezogene Atmungsstörungen (SBAS), insbesondere obstruktive Apnoen und Hypopnoen, können zu Weckreaktionen des zentralen Nervensystems führen. Der resultierende, fragmentierte Schlaf mündet in einer unterschiedlich ausgeprägten Tagesschläfrigkeit, oftmals auch im Sekundenschlaf [1,2]. Weitere langfristige Folgen sind dauerhafte Konzentrationsschwäche, allgemeine körperliche und geistige Einschränkung sowie ein erhöhtes Risiko für Hypertonie, Schlaganfall und kardiovaskuläre Erkrankungen [3-5]. Bei einem Grenzwert für den Apnoe-Hypopnoe-Index (AHI) von 5/h in Kombination mit einer erhöhten Tagesschläfrigkeit liegt die Prävalenz einer SBAS in der Altersgruppe der 30-60-Jährigen bei etwa 2-4\% [6-9]. Bei COPD geben einige Autoren für die Prävalenz für SBAS sogar $10-15 \%$ an $[10,11]$. Andere Autoren sehen keinen Unterschied zur Normalbevölkerung [12].

Die obstruktive Schlafapnoe wird gewöhnlich mit CPAP behandelt $[13,14]$. Eine CPAP-Therapie, die etwa während der Rehabilitation begonnnen wird, führt zu einer Verminderung von Arbeitsunfähigkeitstagen [15]. Daraus ergibt sich eine Kosteneinsparung, die den zusätzlichen Behandlungsaufwand bei weitem übersteigt.

Standardisierte Fragebögen können zur effizienteren Erhebung der Anamnese und somit zu Kostensenkungen beitragen. So zeigt der Berlin-Fragebogen eine hohe Sensitivität und eine annehmbare Spezifität in einem Kollektiv, das sich aus Patienten zusammensetzt, die in Allgemeinkrankenhäusern und allgemeinmedizinischen Praxen behandelt wurden [16,17]. Es wird vermutet, dass der Berlin-Fragebogen bei bestimmten Kollektiven zu einer Überschätzung des OSA-Risikos führt und aufgrund einer erhöhten Anzahl von falsch-positiven oder falsch-negativen Befunden keine Aussagekraft mehr besitzt. Dies könnte bei Patienten zutreffen, deren Tagesschläfrigkeit nicht durch eine OSA, sondern durch eine andere Erkrankung, durch Medikamente oder durch die Lebenssituation verursacht wird. Allgemein findet sich bei Menschen mit schlechtem Gesundheitszustand eine höhere Tagesschläfrigkeit als bei gesunden Menschen [18]. Da diese Symptome maßgeblich in die Bewertung des BerlinFragebogens einfließen, kann dies zu der erwähnten Fehleinschätzung führen. Ziel dieser Studie war es, den prädiktiven Wert des Berlin-Fragebogens in einem Kollektiv von stationären Rehabilitationspatienten mit Atemwegserkrankungen zu untersuchen.

\section{Methoden}

\section{Patienten}

Das Studienkollektiv setzte sich aus 153 Teilnehmern zusammen, die in einer internistisch-pneumologisch ausgerichteten Rehabilitationsklinik (Nordseeklinik Borkum, Deutsche Renten-
Conclusions: The Berlin Questionnaire is a poor predictor of obstructive sleep apnea in a random group of patients undergoing pulmonary rehabilitation.

versicherung Rheinland) untersucht wurden. Die Zuweisung fand durch die Deutsche Rentenversicherung statt (Tab. 1). Über vier Wochen wurde im Rahmen des Rehabilitationsaufenthalts konsekutiv jedem Patienten ein Schlafapnoescreening angeboten. Vor dem Screening mit ApneaLink ${ }^{\circledR}$ (ResMed, München) wurden die Patienten über das Krankheitsbild Schlafapnoe und die gesundheitlichen Folgen aufgeklärt.

\section{Screening}

Für das Screening wurde das Gerät ApneaLink ${ }^{\circledR}$ benutzt, das den Atemfluss über eine Nasenkanüle mittels des Staudrucks misst [23]. Die Daten werden durch eine integrierte Schnittstelle auf einen Personalcomputer übertragen und dort einer automatischen Analyse auf respiratorische Ereignisse unterzogen. Um die Güte dieser automatischen Analyse zu kontrollieren, wurden die Signale am Monitor überprüft. In einem Ergebnisprotokoll wurde unter anderem die Gesamtanzahl der Apnoen und Hypopnoen sowie der RDI festgehalten.

\section{Berlin-Fragebogen}

Die Klassifizierung im Berlin-Fragebogen basiert auf Fragen zu den wichtigsten OSA-Symptomen, die in drei Kategorien unterteilt werden: In der ersten Kategorie werden Schnarchverhalten und während des Schlafes auftretende Atempausen beurteilt, in der zweiten Kategorie wird die Tagesschläfrigkeit bewertet, und in der dritten Kategorie geht es um Übergewicht und Bluthochdruck. Werden mindestens zwei Kategorien positiv bewertet, liegt ein hohes Risiko für eine obstruktive Schlafapnoe vor $[16,17]$.

\section{Epworth-Schläfrigkeitsskala}

Mit der Epworth-Schläfrigkeitsskala wird die Neigung für das Einnicken bzw. Einschlafen in acht typischen Alltagssituationen erfragt. Jeder Frage wird ein Punktwert von 0-3 zugeordnet. Bei einem Gesamtwert größer als 10 auf der Skala von 0 bis 24 liegt ein Verdacht auf eine erhöhte Tagesschläfrigkeit vor [19,20]. Der Fragebogen wurde den Patienten jeweils nach Vergabe der

Tab. 1 Charakteristika des Patientenkollektivs

\begin{tabular}{|c|c|c|c|c|c|}
\hline & $n$ & $\begin{array}{l}\text { Alter } \\
\text { (Jahre) }\end{array}$ & $\begin{array}{l}\text { BMI } \\
\left(\mathrm{kg} / \mathrm{m}^{2}\right)\end{array}$ & $\begin{array}{l}\text { FEV }_{1} \\
\text { (\% SolI) }\end{array}$ & $\begin{array}{l}\text { RDI } \\
(1 / h)\end{array}$ \\
\hline $\begin{array}{l}\text { Asthma } \\
\text { bronchiale }\end{array}$ & 65 & $\begin{array}{l}44,7 \pm \\
10,9\end{array}$ & $\begin{array}{l}28,5 \pm \\
5,8\end{array}$ & $\begin{array}{l}83,9 \pm \\
4,6\end{array}$ & $\begin{array}{c}9,2 \pm \\
10,2\end{array}$ \\
\hline $\begin{array}{l}\text { chronische } \\
\text { Bronchitis }\end{array}$ & 23 & $\begin{array}{l}49,9 \pm \\
9,0\end{array}$ & $\begin{array}{l}29,7 \pm \\
4,7\end{array}$ & $\begin{array}{l}86,6 \pm \\
23,5\end{array}$ & $\begin{array}{l}16,7 \pm \\
21,3\end{array}$ \\
\hline COPD & 55 & $\begin{array}{l}49,3 \pm \\
11,0\end{array}$ & $\begin{array}{l}27,4 \pm \\
5,4\end{array}$ & $\begin{array}{l}71,3 \pm \\
22,7\end{array}$ & $\begin{array}{c}8,5 \pm \\
10,8\end{array}$ \\
\hline $\begin{array}{l}\text { Kombinati- } \\
\text { on Asthma } \\
\text { und COPD }\end{array}$ & 10 & $\begin{array}{l}47,7 \pm \\
6,1\end{array}$ & $\begin{array}{l}26,9 \pm \\
4,9\end{array}$ & $\begin{array}{l}70,3 \pm \\
22,4\end{array}$ & $\begin{array}{l}8,3 \pm \\
9,6\end{array}$ \\
\hline
\end{tabular}


Screeninggeräte um 17 Uhr ausgehändigt und unmittelbar danach ausgefüllt.

\section{Statistische Analyse}

Die individuellen Parameter wurden mit den Methoden der deskriptiven Statistik (Häufigkeit, Mittelwert \pm Standardabweichung, Spanne) behandelt. Sensitivität, Spezifität, positiver und negativer Vorhersagewert, positiver und negativer LikelihoodQuotient für ApneaLink ${ }^{\circledR}$ wurden für RDI-Grenzwerte von 10/h und $15 / h$ berechnet. Häufigkeitsverteilungen wurden mit einem Vierfeldertafeltest und dem $\chi^{2}$-Test auf Unterschiede untersucht. Um zwei Mittelwerte auf Identität zu prüfen, wurde bei Normalverteilung der t-Test, andernfalls der Mann-Whitney-U-Test herangezogen. Beim Vergleich mehrerer Mittelwerte wurde der Kruskal-Wallis-Test durchgeführt. Ein signifikanter Unterschied wurde bei $\mathrm{p}<0,05$ angenommen.

\section{Ergebnisse}

\section{Allgemeine Unterschiede}

In Tab. 1 sind die Charakteristika des Patientenkollektivs dargestellt. Definitionsgemäß fand bei COPD eine Reduktion der $\mathrm{FEV}_{1}$ statt. Bei den 55 Patienten dieser Gruppe handelte es sich gemäß aktueller GOLD-Einteilung (http://goldcopd.com) bei 53\% der Fälle um ein Stadium 1, bei 30\% um ein Stadium 2 und bei $17 \%$ um das Stadium 3.

\section{Geschlechtspezifische Unterschiede}

Im Gesamtkollektiv unterschieden sich die männlichen und weiblichen Studienteilnehmer nicht hinsichtlich Alter (Männer: 47,2 \pm 11,0 Jahre; Frauen: 48,9 \pm 10,6 Jahre) und BMI (Männer: $28,3 \pm 5,6 \mathrm{~kg} / \mathrm{m}^{2}$; Frauen: $28,3 \pm 6,7 \mathrm{~kg} / \mathrm{m}^{2}$ ). Es gab auch keine signifikanten Abweichungen für den BMI $>30 \mathrm{~kg} / \mathrm{m}^{2}$ (Männer: 27,4\%; Frauen: 34,1\%) und für das Vorliegen von Bluthochdruck (Männer: 35,9\%; Frauen: 34,0\%). Die einzelnen Häufigkeiten hinsichtlich der verschiedenen Kategorien und dem erhöhten OSA-Risiko sind in Tab. 2 aufgeführt.

\section{Schlafapnoe-Screening}

Die RDI-Werte der jeweiligen Krankheitsgruppen sind ebenfalls in Tab. 1 aufgeführt. Die Qualität der Aufzeichnungen war durchgängig gut bis sehr gut, wenn die Patienten es geschafft hatten, ApneaLink $^{\circledR}$ einzuschalten. Es gelang 10,5\% der Patienten nicht, das Gerät zu bedienen, woraufhin eine Wiederholung in der nächsten Nacht stattfand. In der zweiten Nacht waren von der Gruppe der Patienten, die das Gerät nicht beim ersten Mal bedienen konnten, 5,2\% abermals nicht in der Lage, ApneaLink ${ }^{\circledR}$ einzuschalten. Einen dritten Anlauf benötigten 1,9\% der Patienten. Zwei Patienten schafften auch dies nicht.
In der Gruppe mit dem gemäß des Berlin-Fragebogens prognostizierten, hohen Risiko für OSA betrug der RDI =12,8 $\pm 16,3 / \mathrm{h}$ (Spanne 1-93/h). In der Gruppe mit niedrigem Risiko war der $\mathrm{RDI}=7,4 \pm 7,8 / \mathrm{h}$ (Spanne 0-33/h). Die RDI-Werte der Gruppen mit hohem und niedrigem Wert waren voneinander signifikant verschieden $(\mathrm{p}<0,05)$. Die Abb. $\bigcirc \mathbf{1} \mathbf{a} \mathrm{u} . \bullet \mathrm{b}$ geben über die jeweiligen RDI-Verteilungen Auskunft.

\section{Epworth-Schläfrigkeitsskala}

Das Gesamtkollektiv wies einen ESS-Wert von 8,8 $\pm 4,8$ auf. Dieser war signifikant verschieden vom Referenzwert 5,7 $\pm 3,0$, der für Gesunde im deutschen Sprachraum angegeben wird [20].

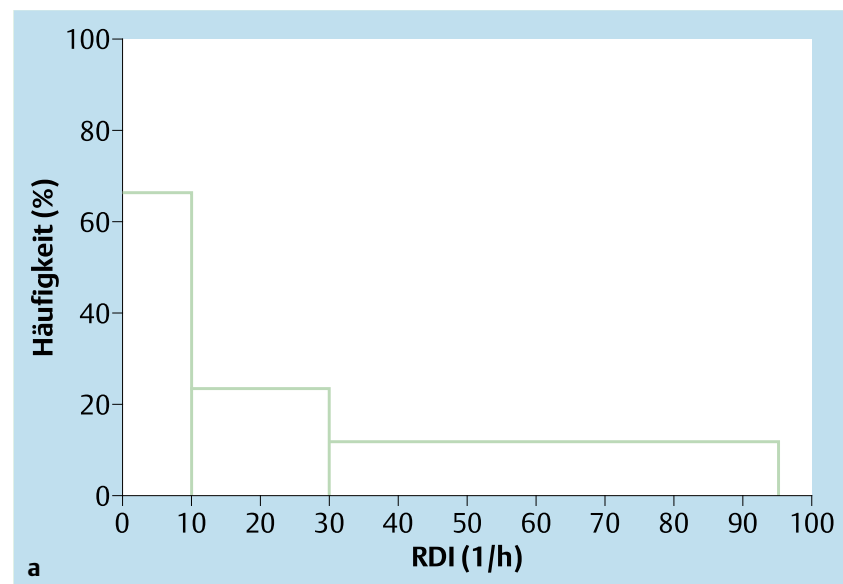

Abb. 1 a Häufigkeit der leichten bis mittelschweren (RDI $=10-30 / \mathrm{h})$ und schweren OSA ( $R D I \geq 30 / h$ ) bei Patienten mit hohem OSA-Risiko.

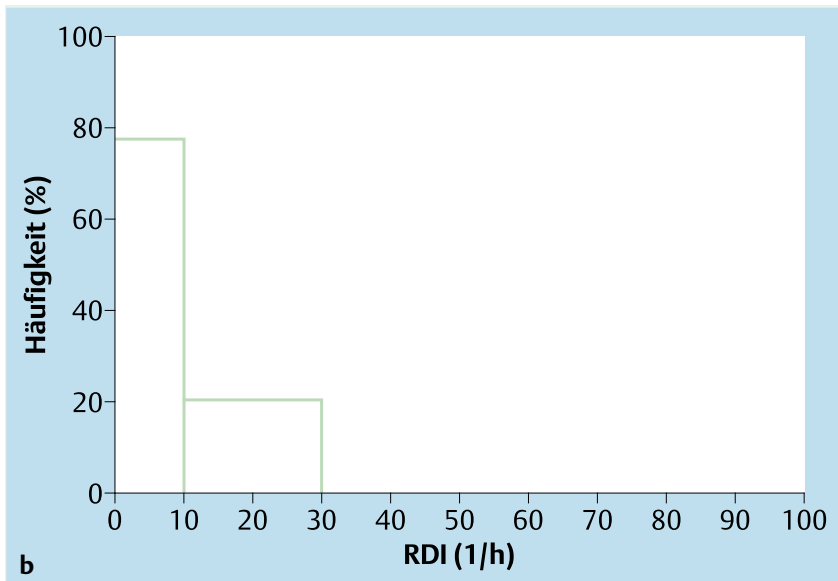

Abb. 1 b Häufigkeit der leichten bis mittelschweren (RDI $=10-30 / h$ ) und schweren OSA (RDI $\geq 30 / h$ ) bei Patienten mit geringem OSA-Risiko.

Tab. 2 Berlin-Fragebogen: Häufigkeit für ein hohes OSA-Risiko, für die verschiedenen Symptome und für ein Einnicken beim Autofahren

\begin{tabular}{|c|c|c|c|c|c|c|c|c|c|c|}
\hline \multirow[b]{2}{*}{ Patienten } & \multicolumn{2}{|c|}{$\begin{array}{l}\text { Hohes Risiko } \\
\text { für OSA }\end{array}$} & \multicolumn{2}{|c|}{$\begin{array}{l}\text { Symptome für } \\
\text { Kategorie } 1\end{array}$} & \multicolumn{2}{|c|}{$\begin{array}{l}\text { Symptome für } \\
\text { Kategorie } 2\end{array}$} & \multicolumn{2}{|c|}{$\begin{array}{l}\text { Symptome für } \\
\text { Kategorie } 3\end{array}$} & \multicolumn{2}{|c|}{$\begin{array}{l}\text { Beim Autofahren } \\
\text { eingenickt }\end{array}$} \\
\hline & $\%$ & $n$ & $\%$ & $\mathbf{n}$ & $\%$ & $\mathbf{n}$ & $\%$ & $\mathbf{n}$ & $\%$ & $n$ \\
\hline$m(n=106)$ & 50,9 & 54 & $64,2^{*}$ & 68 & 39,7 & 41 & 50,9 & 54 & 19,8 & 21 \\
\hline$w(n=47)$ & 48,9 & 23 & $42,6^{*}$ & 20 & 42,6 & 20 & 51,1 & 24 & 10,6 & 5 \\
\hline Gesamt $(n=153)$ & 50,3 & 77 & 57,5 & 88 & 39,9 & 61 & 51,0 & 74 & 17,0 & 26 \\
\hline
\end{tabular}

${ }^{*} \mathrm{p}<0,05$ 
Tab. 3 ESS-Werte für die Gruppe mit und ohne SBAS bei einem RDI-Grenzwert von 10/h, für die verschiedenen Krankheitsgruppen, für Männer und Frauen

\begin{tabular}{lccl} 
& $\mathbf{n}$ & ESS & Spanne \\
$\mathrm{RDI}<10 / \mathrm{h}$ & 105 & $8,6 \pm 4,6$ & $0-21$ \\
\hline $10 / \mathrm{h} \geq \mathrm{RDI}$ & 35 & $8,3 \pm 5,0$ & $0-19$ \\
$<30 / \mathrm{h}$ & & $10,9 \pm 5,1^{*}$ & $2-17$ \\
$\mathrm{RDI} \geq 30 / \mathrm{h}$ & 13 & $8,8 \pm 4,6$ & $0-19$ \\
\hline Männer & 106 & $8,7 \pm 5,1$ & $0-21$ \\
\hline Frauen & 47 & $9,1 \pm 4,9$ & $1-21$ \\
\hline Asthma & 65 & $9,3 \pm 4,8$ & $1-19$ \\
chronische & 23 & & \\
Bronchitis & & $8,0 \pm 4,7$ & $0-16$ \\
COPD & 55 &
\end{tabular}

* $\mathrm{p}<0,1$ gegenüber der Gruppe ohne SBAS (RDI $<10 / \mathrm{h})$ und der Gruppe mit leichter bis mittelschwerer SBAS $(10 / \mathrm{h} \geq \mathrm{RDI}<30 / \mathrm{h})$

Alle Untergruppen (Asthma, chronische Bronchitis oder COPD) wiesen einen ESS-Wert auf, der ebenfalls signifikant vom Referenzwert der Gesunden verschieden war (Tab. 3). Der höchste ESS-Wert fand sich bei schwerer OSA (RDI $\geq 30 / h$ ). Dieser unterschied sich von den ESS-Werten für Patienten ohne ( $p<0,1)$ oder einer leichten OSA $(p<0,1)$. Patienten, die angaben, schon einmal beim Autofahren eingenickt zu sein, waren durch einen ESSWert von 11,6 $\pm 5,1$ (Spanne 3-21) gekennzeichnet, der signifikant größer war als der ESS-Wert des gesamten Studienkollektivs und als in den anderen Untergruppen.

\section{Berlin-Fragebogen: Sensitivität, Spezifität,}

Vorhersagewerte und Likelihood-Quotienten

Sensitivität und Spezifität des Berlin-Fragebogens als Indikator für ein prognostiziertes, hohes OSA-Risiko hängen davon ab, welche Grenzwerte für den RDI gewählt werden (Tab. 4). Für Patienten mit einem prognostizierten, hohen OSA-Risiko lag die Sensitivität bei einem Grenzwert von RDI $=10 / \mathrm{h}$ bei $62,5 \%$ und die Spezifität bei $53,8 \%$. Der positive Vorhersagewert betrug $38,4 \%$ und der negative $74,2 \%$. Der positive Likelihood-Quotient wies einen Wert von 1,35 auf und der negative einen Wert von 0,69. Bei Anhebung des Grenzwertes auf RDI=15/h änderten sich Sensitivität, Spezifität, positiver und negativer Vorhersagewert sowie positiver und negativer Likelihood-Quotient nicht signifikant.

\section{Diskussion}

Diese Studie zeigte, dass der Berlin-Fragebogen bei Rehabilitationspatienten mit Atemwegserkrankungen kein geeignetes Instrument zur Risikostratifizierung einer obstruktiven Schlafapnoe darstellt. Für einen akzeptablen Screeningtest fordern wir für unsere Studie, dass er eine Sensitivität von mindestens $75 \%$

Tab. 4 Sensitivität, Spezifität, positiver und negativer Vorhersagewert, positiver und negativer Likelihood-Quotient des Berlin-Fragebogens im Vergleich zu verschiedenen Grenzwerten des RDI von ApneaLink ${ }^{\circledR}$

\begin{tabular}{|c|c|c|c|c|c|c|}
\hline $\begin{array}{l}\text { RDI } \\
(1 / h)\end{array}$ & $\begin{array}{l}\text { Sensiti- } \\
\text { vität (\%) }\end{array}$ & $\begin{array}{l}\text { Spezifi- } \\
\text { tät (\%) }\end{array}$ & $\begin{array}{l}\text { PPV } \\
(\%)\end{array}$ & $\begin{array}{l}\text { NPV } \\
(\%)\end{array}$ & LR+ & LR- \\
\hline 10 & 62,5 & 53,8 & 38,4 & 74,2 & 1,35 & 0,69 \\
\hline 15 & 67,2 & 52,8 & 25,1 & 84,7 & 1,42 & 0,62 \\
\hline
\end{tabular}

und eine Spezifität von mindestens 70\% aufweisen sollte. Diese Kriterien sind für den Berlin-Fragebogen in dem hier untersuchten Patientenkollektiv nicht erfüllt. Bei einem Grenzwert von 10/h für den RDI betrug die Sensitivität des Berlin-Fragebogens hinsichtlich eines hohen OSA-Risikos lediglich 62,5\%, und die Spezifität lag nur bei 53,8\%. Der positive Vorhersagewert - also die Wahrscheinlichkeit dafür, dass ein Patient, dem der BerlinFragebogen ein hohes OSA-Risiko zuordnet, tatsächlich eine OSA aufweist - lag lediglich bei $38 \%$. Auch der positive Likelihood-Quotient, der angibt, wie viel häufiger der Berlin-Fragebogen einem OSA-Patienten ein hohes OSA-Risiko zuweist als einem Patienten ohne OSA, fiel mit einem Wert von 1,35 gering aus. In diesem Kollektiv von Rehabilitationspatienten mit einem durchschnittlichen Alter von 47,9 \pm 10,3 Jahren besitzt der Berlin-Fragebogen daher keine Aussagekraft, die über ein zufälliges Urteil hinausgeht. Auch durch Anheben des RDI-Grenzwertes auf 15/h konnte die diagnostische Zuverlässigkeit des Berlin-Fragebogens nicht verbessert werden. Die Sensitivität stieg nur wenig auf $67,2 \%$ an. Zwar sinkt die Sensitivität gewöhnlich, aber in dieser Studie liegt der Fall anders: Je höher der RDI-Grenzwert für den Verdacht auf OSA liegt, desto geringer ist die Anzahl der Betroffenen, denen gleichzeitig der Berlin-Fragebogen ein erhöhtes OSA-Risiko zuweist. Die Gruppe der richtig-positiven Ergebnisse nimmt also zahlenmäßig ab. Andererseits sinkt auch der Anteil der falsch-negativen Ergebnisse, d. h. wenn der BerlinFragebogen kein erhöhtes Risiko in der Gruppe des erhöhten RDI-Grenzwerts aufweist. Deshalb kann es dazu kommen, dass die Sensitivität bei Erhöhen des Grenzwertes steigt, statt wie gewöhnlich zu fallen.

Die unzureichende Aussagekraft des Berlin-Fragebogens ergab sich unabhängig von der Erkrankung, vom Alter, vom BMI und vom Geschlecht. In den unterschiedlichen Kategorien des BerlinFragebogens gab es lediglich bei Schnarchen und Atempausen signifikante, geschlechtsspezifische Unterschiede: Bei Männern traten diese Merkmale häufiger auf als bei Frauen.

Die Ergebnisse dieser Studie unterschieden sich von zwei Referenzstudien, deren Teilnehmer sich in Allgemeinkrankenhäusern und in allgemeinmedizinischen Praxen in Behandlung befanden $[16,17]$. Alle Symptome und Risikofaktoren traten bei den Rehabilitationspatienten signifikant häufiger auf als im Referenzkollektiv. Schnarchen und Atempausen waren bei Männern um 6,7\% häufiger anzutreffen, bei Frauen um 12,6\%. Das Symptom Tagesschläfrigkeit kam bei Männern um 19,0\% häufiger vor, bei Frauen um 14,6\%. Bluthochdruck und Übergewicht waren bei Männern um 6,1\% häufiger anzutreffen, bei Frauen um $11,1 \%$.

Auch die Einschätzung des OSA-Risikos steht im Gegensatz zu den zwei Referenzstudien, die den Berlin-Fragebogen hinsichtlich seiner diagnostizierten Zuverlässigkeit evaluierten. Die wesentliche Ursache für diesen scheinbaren Widerspruch liegt darin, dass die Patienten unserer Studie im Vergleich zu den Referenzstudien über eine ausgeprägtere Tagesschläfrigkeit berichteten. Die erhöhten ESS-Werte erklärten sich wahrscheinlich durch den schlechteren Gesundheitszustand der chronisch kranken Rehabilitationspatienten [18]. Eine Rolle spielte möglicherweise auch die Klimaänderung bzw. das Seeklima, das bekanntlich zu Beginn eines Aufenthalts am Meer zu einer vorübergehend erhöhten Schläfrigkeit führen kann. Diese Möglichkeit lässt sich bei ähnlichen Studien in Zukunft ausschließen, indem die Befragung auf Schläfrigkeit am Ankunftstag des Rehabilitationsaufenthalts durchgeführt wird. Die erhöhten ESS-Werte könnten im Einzelfall auch auf eine Überbewertung der indivi- 
duellen Schläfrigkeit zurückzuführen sein, um ein eventuell vorhandenes Rentenbegehren in die gewünschten Bahnen zu lenken. Außerdem könnte die Einnahme von Medikamenten mit einer erhöhten Schläfrigkeit einhergehen. Die hohe Tagesschläfrigkeit führt dazu, dass die zweite Kategorie des Berlin-Fragebogens übermäßig oft positiv gewertet wird. Wenn nun ein Patient, der keine OSA aufweist, dafür aber unter Bluthochdruck, Übergewicht oder Schnarchen leidet, wird ihm fälschlicherweise ein hohes OSA-Risiko zugeordnet.

Obwohl der Atmungsmonitor ApneaLink ${ }^{\circledR}$ leicht zu bedienen ist, waren beim ersten Versuch immerhin 10,5\% der Patienten nicht in der Lage, das Gerät ordnungsgemäß anzulegen bzw. einzuschalten. Diese Quote konnte auf 1,3\% gesenkt werden, indem bei den Patienten das nächtliche Screening bis zu dreimal wiederholt wurde. Die Ursachen der ermittelten Fehlrate beruhen auf mangelnder Konzentration beim Einschalten, Nervosität, Unaufmerksamkeit bei der Einweisung in die Bedienung des Gerätes oder geistiger Limitation. Auch absichtliche Fehlbedienung bei Patienten mit einem Rentenbegehren stellt eine Möglichkeit dar. Die Fehlerrate kann erniedrigt werden, indem man jeden Patienten einzeln einweist und er sich unter Aufsicht mit dem Gerät vertraut macht oder das Klinikpersonal im Nachtdienst die Aufzeichnung startet. Da nicht beliebig viel Zeit zur Verfügung stand, wurde in dieser Studie generell auf eine persönliche Anleitung verzichtet, und es fand eine Einweisung in einem Vortrag vor etwa 20 Patienten statt. Anschließend wurde ein Freiwilliger gebeten, das Gerät anzulegen und zu bedienen. Nach der Einweisung schafften es alle Freiwilligen im ersten Versuch, das Gerät fehlerfrei ein- und auszuschalten.

In diesem Studienkollektiv gaben $17 \%$ der Patienten an, dass sie schon mindestens einmal beim Autofahren eingenickt waren. Dies traf vor allen Dingen bei Patienten mit einem hohen ESSWert zu - unabhängig vom Schweregrad der OSA. Es lässt sich daher verallgemeinern, dass diese Gefahr nicht nur bei Schlafapnoikern anzutreffen ist, sondern bei allen Patienten, die unter erhöhter Tagesschläfrigkeit leiden. Der behandelnde Arzt sollte deshalb einen Patienten mit erhöhter Tagesschläfrigkeit und einer beliebigen, chronischen Erkrankung, die den allgemeinen Gesundheitszustand herabsetzt, auf das mögliche Risiko des Sekundenschlafs beim Autofahren aufmerksam machen.

Als weiteres Ergebnis belegte diese Studie, dass sich die Tagesschläfrigkeit von Rehabilitationspatienten mit Atemwegserkrankungen ohne $(\mathrm{RDI}<10 / \mathrm{h})$ oder mit nur leichter bis mittelschwerer OSA $(\mathrm{RDI}=10-29 / \mathrm{h})$ nicht unterscheidet. Erst bei schwerer OSA (RDI $\geq 30 / \mathrm{h})$ findet sich eine höhere Tagesschläfrigkeit als bei Patienten ohne oder mit nur leichter bis mittelschwerer OSA. Die Streuungsbreite ist jedoch hoch, so dass am Schweregrad der OSA nicht zwangsläufig auf das Ausmaß der Tagesschläfrigkeit geschlossen werden kann, sondern der Einsatz der ESS generell empfohlen wird.

Als Goldstandard der Diagnostik einer OSA und Schweregradbeurteilung des AHI gilt die Polysomnographie [21]. In dieser Arbeit wurde zur Quantifizierung der respiratorischen Ereignisse als Screeningmethode ApneaLink ${ }^{\circledR}$ eingesetzt. Validierungsuntersuchungen haben eine gute Übereinstimmung des RDI mit dem durch eine Polysomnographie ermittelten AHI ergeben [23]. Da die Kenngrößen des ApneaLink ${ }^{\circledR}$ bekannt sind, ist nach Einführung geeigneter Korrekturfaktoren deshalb davon auszugehen, dass sich die Schweregradeinteilung der Schlafapnoe in dieser Studie nicht relevant von der Zuordnung auf der Basis einer Polysomnographie unterscheidet. Aus diesem Grunde bietet sich ein kostengünstiges Atmungsmonitoring (z.B. ApneaLink ${ }^{\circledR}$ ) an, das ohne signifikanten Mehraufwand eine hohe Prätestwahrscheinlichkeit für das Vorliegen oder den Ausschluss einer klinisch relevanten, obstruktiven Schlafapnoe in der Polysomnographie ermöglicht.

Zusammenfassend lässt sich feststellen, dass der Berlin-Fragebogen bei Patienten mit chronischen Atemwegserkrankungen in der pneumologischen Rehabilitation kein geeignetes Instrument zur Identifizierung eines hohen Risikos für das Vorliegen einer klinisch relevanten, obstruktiven Schlafapnoe als Komorbidität darstellt. Aufgrund der hohen Anzahl falsch positiver und falsch negativer Ergebnisse trägt der Berlin-Fragebogen unter diesen Rahmenbedingungen nicht zur Erhöhung der Prätestwahrscheinlichkeit einer obstruktiven Schlafapnoe bei.

\section{Anmerkung}

Mit Unterstützung der Gesellschaft für Rehabilitationsforschung (GfR), der Deutschen Rentenversicherung Rheinland (DRV) und der Arbeitsgemeinschaft zur Förderung der Pneumologie an der Ruhrlandklinik (AFPR).

\section{Literatur}

1 Teran-Santos J, Jiminez-Gomez A, Cordero-Guevara J: The association between sleep apnea and the risk of traffic accidents: Cooperative Group Burgos-Santander. N Engl J Med 1999; 340: 847-851

2 Lyznicki JM, Doege TC, Davis RM et al: Sleepiness, driving, and motor vehicle crashes. Council on Scientific Affairs, American Medical Association. JAMA 1998; 279: 1908 - 1913

3 Ulfberg J, Carter N, Talback $M$ et al: Excessive daytime sleepiness at work and subjective work performance in the general population and among heavy snorers and patients with obstructive sleep apnea. Chest 1996; 110: 659-663

4 Peppard PE, Young T, Palta $M$ et al: Prospective study of the association between sleep-disordered brerathing and hypertension. $\mathrm{N}$ Engl J Med 2000; 342: 1378 - 1384

5 Marin JM, Carrizo SJ, Vicente E et al: Long-term cardiovascular outcomes in men with obstructive sleep apnoea-hypopnoea with or without treatment with continuous positive airway pressure: an observational study. Lancet 2005; 365: 1046-1053

6 Young T, Palta M, Dempsey J et al: The occurrence of sleep-disordered breathing among middle-aged adults. N Eng J Med 1993; 328: 1230 1235

7 Marin JM, Gascon JM, Carrizo S et al: Prevalence of sleep apnea syndrome in the Spanish adult population. Int J Epidemiol 1997; 26: $381-386$

8 Olson LG, King MT, Hensley MJ et al: A community study of snoring and sleep disordered breathing. Prevalence. Am J Respir Crit Care Med 1995; 152 : 711 - 716

9 Ohayon MM, Guilleminault C, Priest RG et al: Snoring and breathing pauses during sleep: telephone interview survey of a United Kingdom population sample. BMJ 1997; 314: 860-863

10 Chaouat A, Weitzenblum E, Krieger J et al: Association of chronic obstructive pulmonary disease and sleep apnea syndrome. Am J Respir Crit Care Med, 1995; 151 (1): 82-86

11 Sanders $M H$, Newman $A B$, Haggerty $C L$ et al: Sleep Heart Health Study. Sleep and sleep-disordered breathing in adults with predominantly mild obstructive airway disease. Am J Respir Crit Care Med 2003; 167: 7-14

12 Bednarek M, Plywaczewski R, Jonczak L et al: There is no relationship between chronic obstructive pulmonary disease and obstructive sleep apnea syndrome: a population study. Respiration 2005; 72: 142-149

13 Fischer J, Raschke F: Economic and medical significance of sleep-related breathing disorders. Respiration 1997; 64 Suppl 1: 39- 44

14 Quan SF, Howard BV, Iber C et al: The Sleep Heart Health Study: design, rationale, and methods. Sleep 1997; 20: 1077 - 1085

15 Fischer J, Raschke F: Kosten-Nutzen-Analyse bei Patienten mit schlafbezogenen Atmungsstörungen. Biomed Tech (Berl) 2003; 48: 245 251 
16 Netzer NC, Stoohs RA, Netzer CM et al: Using the Berlin Questionnaire to identify patients at risk for the sleep apnea syndrome. Ann Intern Med 1999; 131: 485-491

17 Netzer NC, Hoegel JJ, Loube D et al: Prevalence of symptoms and risk of sleep apnea in primary care. Chest 2003; 124: 1406 - 1414

18 Briones B, Adams $N$, Strauss $M$ et al: Relationship between sleepiness and general health status. Sleep 1996; 19: 583-588

19 Johns MW: A new method for measuring daytime sleepiness: the Epworth sleepiness scale. Sleep 1991; 14: 540-545

20 Bloch KE, Schoch OD, Zhang JN et al: German version of the Epworth Sleepiness Scale. Respiration 1999; 66: 440-447

21 Fischer J, Mayer G, Peter JH (Hrsg): Nicht-erholsamer Schlaf - Leitlinie S2 der Deutschen Gesellschaft für Schlafforschung und Schlafmedizin. Berlin, Wien: Blackwell Verlag, 2001

22 Hein H, Raschke F, Köhler D et al: Leitlinie zur Diagnostik und Therapie schlafbezogener Atmungsstörungen beim Erwachsenen - Leitlinien der Deutschen Gesellschaft für Pneumologie in Zusammenarbeit mit der Deutschen Gesellschaft für Schlafforschung und Schlafmedizin. Pneumologie 2001; 55: 339-342

23 Wang $Y$, Teschler $T$, Weinreich $G$ et al: Validierung von microMESAM als Screeningsystem für schlafbezogene Atmungsstörungen. Pneumologie 2003; 57: 734- 740

24 Rowley JA, Aboussouan LS, Badr MS: The use of clinical prediction formulas in the evaluation of obstructive sleep apnea. Sleep 2000; 23: 929-938

25 Harding SM: Prediction formulae for sleep-disordered breathing. Curr Opin Pulm Med 2001; 7: 381 - 385

26 Zweig MH, Campbell G: Receiver-operating characteristic (ROC) plots: a fundamental evaluation tool in clinical medicine. Clin Chem 1993; 39: $561-577$

27 Jaeschke R, Guyatt G, Sackett DL for the Evidence-Based Medicine Working Group: Users' Guides to the Medical Literature. II How to use an article about a diagnostic test. $\mathrm{B}$. What are the results and will they help me in caring for my patient? JAMA 1994; 271: $703-707$

\section{Leserbrief}

\section{Wenzel et al}

Vergleich der unspezifischen bronchialen Provokation mit Metacholin unter kontrollierter und freier Inhalation

Pneumologie 2006; 60: $467-471$

Sehr geehrte Herren Professoren, sehr geehrte Damen und Herren,

zweifellos stellen das APS von Jaeger-Viasys wie auch das AKITASystem von Activaero geeignete Instrumente für eine aussagekräftige bronchiale Provokation dar.

Das in dem Beitrag diskutierte Fehlen eines „Gold-Standards“, der kontrovers diskutierte diagnostische und prognostische Wert dieser Untersuchung wie auch die nicht optimale Übereinstimmung beider Methoden werfen aus meiner Sicht die Frage auf, ob deshalb so kostspielige Apparate und komplizierte Untersuchungsabläufe erforderlich sind.

Diese von Subspezialisten mit dem Ziel einer besseren Standardisierung der unspezifischen bronchialen Provokation entwickelten Verfahren sind von der Medizingeräteindustrie natürlich interessiert aufgenommen und umgesetzt worden - und nun wird einer (vermeintlich?) zahlungskräftigen Ärzteschaft ein weiteres teueres Gerät offeriert.

$\mathrm{Zu}$ Zeiten meiner pneumologischen Grundausbildung vor ca. 20 Jahren wurde an einer renommierten pneumologischen Fachklinik die unspezifische bronchiale Provokation mit Metacholinlösung definierter Konzentration durchgeführt, von der der Patient mittels eines handelsüblichen Pari-Düsenverneblers zunächst 1 Atemzug inhalierte mit Verdopplung der Atemzüge bis zum Eintreten der Obstruktion oder Erreichen von 16 Atemzügen, nach jeder „Konzentrationsstufe“ eine komplette Lungenfunktion mit Raw und FEV1.

Damit waren stets eindeutige Ergebnisse zu erzielen, die auch gutachterlichen Fragestellungen standhielten. In meiner weiteren Tätigkeit als Pneumologe einer kleineren internistischen Abteilung habe ich das so beibehalten. Ob dieses Verfahren allerdings den Erfordernissen der KV-Abrechnung entspricht, weiß ich als Klinikarzt nicht. Sicherlich gibt es aber mit geringem apparativem (und damit finanziellem) Aufwand und erträglichem organisatorischem Aufwand wertvolle diagnostische Hinweise.

Mit freundlichen Grüßen

Dr. med. Peter Pommer

Bayerwaldklinik

Klinikstr. 22

93413 Cham-Windischbergerdorf

E-mail: pommer@bayerwaldklinik.de 